\title{
HIGH-RESOLUTION INFRARED IMAGING OF THE COMPACT NUCLEAR SOURCE IN NGC 4258
}

\author{
R. Chary, ${ }^{1,2}$ E. E. Becklin, ${ }^{1}$ A. S. Evans, ${ }^{3}$ G. Neugebauer, ${ }^{4}$ N. Z. Scoville, ${ }^{3}$ K. Matthews, ${ }^{4}$ and M. E. Ressler ${ }^{5}$ \\ Received 1999 May 5 ; accepted 1999 October 26
}

\begin{abstract}
We present high-resolution imaging of the nucleus of NGC 4258 from 1 to $18 \mu \mathrm{m}$. Our observations reveal that the previously discovered compact source of emission is unresolved even at the near-infrared resolution of $\sim 0$ ".2 FWHM, which corresponds to about $7 \mathrm{pc}$ at the distance of the galaxy. This is consistent with the source of emission being the region in the neighborhood of the purported $3.5 \times 10^{7} M_{\odot}$ black hole. After correcting for about 18 mag of visual extinction, the infrared data are consistent with an $F_{v} \propto v^{-1.4 \pm 0.1}$ spectrum from 1.1 to $18 \mu \mathrm{m}$, implying a nonthermal origin. Based on this spectrum, the total extinction-corrected infrared luminosity $(1-20 \mu \mathrm{m})$ of the central source is $2 \times 10^{8} L_{\odot}$. We argue that the infrared spectrum and luminosity of the central source obviates the need for a substantial contribution from a standard, thin accretion disk at these wavelengths and calculate the accretion rate through an advection-dominated accretion flow to be $\dot{M} \sim 10^{-3} M_{\odot} \mathrm{yr}^{-1}$. The agreement between these observations and the theoretical spectral energy distribution for advection-dominated flows provides evidence for the existence of an advection-dominated flow in this low-luminosity active galactic nucleus.
\end{abstract}

Subject headings: accretion, accretion disks - galaxies: individual (NGC 4258) - galaxies: nuclei infrared: general - radiation mechanisms: nonthermal

\section{INTRODUCTION}

NGC 4258 (M106) is a weakly active Seyfert 1 galaxy (Filippenko 1996) at a distance of $\sim 7 \mathrm{Mpc}$ (we adopt $H_{0}=$ $65 \mathrm{~km} \mathrm{~s}^{-1} \mathrm{Mpc}^{-1}$; at this distance $\left.1^{\prime \prime}=33.9 \mathrm{pc}\right)$. The nucleus of the galaxy is a prototypical low-luminosity AGN. Dynamical evidence for the existence of a massive black hole in the nucleus comes from high spatial resolution VLBI observations of water masers, which reveal the existence of a thin edge-on disk of inner radius 4 mas and outer radius 8 mas (Miyoshi et al. 1995). The high-velocity features of the masers were found to be symmetrically offset from the systemic velocity by $700-1000 \mathrm{~km} \mathrm{~s}^{-1}$, as would be expected in a Keplerian disk. The inferred binding mass of $3.5 \pm 0.1 \times 10^{7} M_{\odot}$ within a radius of $0.14 \mathrm{pc}$ (Herrnstein, Greenhill, \& Moran 1996) makes it the strongest supermassive black hole candidate after the nucleus of the Galaxy (Genzel et al. 1997, Ghez et al. 1998). The adopted binding mass and distance for the galaxy are consistent with the more recent measurements of Herrnstein et al. (1999). Observations by the X-ray satellite $A S C A$ indicate the existence of a strongly obscured source with a photon index of $\Gamma=1.8 \pm 0.3$ at the nucleus of the galaxy (Makishima et al. 1994). The photon index $\Gamma=1+\gamma$, where $\gamma$ is the spectral index. The line-of-sight column density of $N_{\mathrm{H}}=1.5 \times 10^{23}$ $\mathrm{cm}^{-2}$, derived from a fit to the absorbed X-ray data, corresponds to $A_{V} \sim 80 \mathrm{mag}$, which would obscure the nucleus at visible wavelengths. The extinction-corrected $\mathrm{X}$-ray luminosity from 2 to $10 \mathrm{keV}$ is $4 \times 10^{40} \mathrm{ergs} \mathrm{s}^{-1}$ and is relatively

\footnotetext{
${ }^{1}$ Division of Astronomy and Astrophysics, University of California, Los Angeles, Los Angeles, CA 90095-1562.

${ }^{2}$ Present address: Department of Astronomy and Astrophysics, University of California, Santa Cruz, Santa Cruz, CA 95064; rchary@ucolick.org.

${ }^{3}$ Owens Valley Radio Observatory, California Institute of Technology, Pasadena, CA 91125.

${ }^{4}$ Palomar Observatory, California Institute of Technology, Pasadena, CA 91125.

${ }^{5}$ Jet Propulsion Laboratory, Pasadena, CA 91109.
}

insensitive to the absorption column density. Based on arguments that the X-ray luminosity is typically about $5 \%-40 \%$ of the bolometric luminosity in AGNs (Mushotzky, Done, \& Pounds 1993), $L_{\mathrm{Bol}}$ for the central engine in NGC 4258 is of order $10^{41} \mathrm{ergs} \mathrm{s}^{-1}$. This is $\sim 2$ $\times 10^{-5} L_{\mathrm{E}}$, where $L_{\mathrm{E}}$ is the Eddington luminosity of the massive black hole at the nucleus and corresponds to $4.4 \times 10^{45} \mathrm{ergs} \mathrm{s}^{-1}$.

The low bolometric luminosity of the central engine can be explained by an incorrect estimation of $L_{\mathrm{Bol}}$, since the overall spectral energy distribution of the central engine is not well known. Alternatively, since $L_{\mathrm{Bol}}=\eta \dot{M} c^{2}$, where $\eta$ is the radiative efficiency and $\dot{M}$ is the mass accretion rate of the central engine, a low bolometric luminosity could be attributed to (1) a sub-Eddington mass accretion rate through a standard, geometrically thin disk; for the black hole in NGC 4258, the Eddington mass accretion rate $\dot{M}_{\mathrm{E}} \sim 0.75 M_{\odot} \mathrm{yr}^{-1}$, where $\eta$ has a fiducial value of $10 \%$; or (2) a low radiative efficiency for the accretion phenomenon whereby most of the viscously generated energy is carried through the event horizon of the black hole. This is more commonly called an advection-dominated accretion flow (ADAF; Narayan \& Yi 1995).

Strong line-of-sight extinction to the nucleus of NGC 4258 makes estimating the intrinsic spectral energy distribution difficult. Wilkes et al. (1995; hereafter W95) detected a polarized continuum with $F_{v} \propto v^{-1.1 \pm 0.2}$ in scattered light at visible wavelengths. The luminosity of the central source is only weakly constrained by these observations to lie in the range $10^{41}-10^{43} \mathrm{ergs} \mathrm{s}^{-1}$ and is dependent on the scattering model. Infrared observations are less susceptible to extinction effects and are therefore a better probe of the energy spectrum and the output luminosity. Chary \& Becklin (1997, hereafter CB97) detected a compact infrared source in the nucleus of the galaxy. Based on the visible light polarimetric observations of W95, CB97 concluded that the intrinsic spectrum of the infrared source is a power law $\left(F_{v} \propto v^{-1}\right)$ and that the observed $J-H, H-K$ colors could be explained by about 17 mag of visual extinction 
along the line of sight. While the absolute extinctioncorrected luminosity at these wavelengths is consistent with an ADAF model (Lasota et al. 1996), the derived spectrum poses some problems because at these wavelengths, both the ADAF model and the standard thin disk model predict a $v^{1 / 3}$ Comptonized blackbody spectrum arising from a cool geometrically thin, optically thick disk (Pringle 1981). In addition, infrared emission from thermal reprocessing of the central visible/UV continuum by dust in a flared disk or "torus" could not be unambiguously ruled out. The only observation at mid-infrared wavelengths that can potentially trace the dust distribution or constrain the overall spectrum of the central source is the $8-13 \mu \mathrm{m} \mathrm{5"7}$ diameter data from Rieke \& Lebofsky (1978; hereafter RL78). This measurement could be contaminated by extended emission from regions of nuclear star formation.

Lasota et al. (1996) applied an ADAF model to fit the X-ray spectrum of NGC 4258. The model consists of an inner, optically thin flow that is geometrically thick. The ADAF exists from an inner radius of $3 R_{\mathrm{S}}$ up to a radius of $10-100 R_{\mathrm{S}}$, where $R_{\mathrm{S}}$ is the Schwarzschild radius of the central black hole and corresponds to $3.4 \times 10^{-6} \mathrm{pc}$. The ions that are heated by the viscously generated energy have a temperature of $\sim 10^{12} \mathrm{~K}$, but their radiative timescale is longer than the accretion timescale, resulting in most of the energy being transported into the event horizon of the black hole by radial convection or "advection." The electrons $\left(T_{e} \sim 10^{9} \mathrm{~K}\right)$ produce synchrotron photons that could be the seeds for self-Comptonization to produce a hard X-ray spectrum. The model also includes a cooler geometrically thin, optically thick disk that extends up to $10^{6} R_{\mathrm{S}}$ to reproduce the blackbody bump seen at visible/UV wavelengths in most AGNs. This kind of accretion flow allows a "normal" accretion rate of $\dot{m} \sim 0.2 \alpha$ but requires a low radiative efficiency since most of the viscously generated energy is transported into the event horizon of the black hole by the advection process. Here, $\alpha \leq 1$ is the dimensionless viscosity parameter defined by Shakura \& Sunyaev (1973), while $\dot{m}=\dot{M}\left(M_{\odot} \mathrm{yr}^{-1}\right) / \dot{M}_{\mathrm{E}}$ is the accretion rate in units of the Eddington rate. Mahadevan (1997, hereafter M97) has investigated the spectral energy distribution from an ADAF in detail and shown that the Comptonization of soft cyclosynchrotron photons results in a power-law spectrum between $10^{12}$ and $10^{17} \mathrm{~Hz}$. The spectral index of the power law is essentially constrained by the mass accretion rate through the ADAF.

In this paper, we present high-resolution imaging of the nucleus of NGC 4258 at mid-infrared and near-infrared wavelengths. The observations place a better handle on the spectrum and bolometric luminosity of the central engine. This allows us to constrain the mass accretion rate $\dot{M}$ of the black hole based on the different accretion models that have been proposed.

\section{OBSERVATIONS AND RESULTS}

The 1.1-2.2 $\mu \mathrm{m}$ data were obtained using the NearInfrared Camera and Multi-Object Spectrometer (NICMOS) Camera 2 on the Hubble Space Telescope (HST; Thompson et al. 1998). The camera has a $256 \times 256$ $\mathrm{HgCdTe}$ array with pixel scales of $00^{\prime \prime} 0762$ and 0.0755 pixel $^{-1}$ in $x$ and $y$, respectively, providing a $\sim 19^{\prime \prime} .5 \times 19^{\prime \prime} .3$ field of view. Frames were taken in three filters, F110W, F160W, and F222M, which correspond to 1.1, 1.6, and 2.2 $\mu \mathrm{m}$, respectively. Observations of the galaxy were made using a four-point spiral dither in each filter setting; the offset per dither position was 25.5 pixels (1".9). Nondestructive reads (MULTIACCUM) were obtained at each dither position, with integration times of 40 (F110W, F160W) and $56(\mathrm{~F} 222 \mathrm{M}) \mathrm{s}$ per position. The total integration times were thus 160 (F110W, F160W) and 224 (F222M) s. In addition to the galaxy observations, blank-sky observations were done with the F222M filter to determine the background level at $2.2 \mu \mathrm{m}$, and dark frames were taken using the same MULTIACCUM sequences as the galaxy observations.

The data were reduced with IRAF. The dark was first created, then the NICMOS data were dark subtracted, flatfielded, and corrected for cosmic rays using the IRAF pipeline reduction routine CALNICA (Bushouse 1997; see Scoville et al. 2000 for details about the reduction procedure). The calibrated images contained pixels with reduced quantum efficiency due to contaminants on the array surface; thus, a mask was created to minimize their effect. The coronographic hole and column 128 were also masked on all images; the latter feature was masked because of its sensitivity to minute discrepancies in dark subtraction (i.e., the dark current rises sharply toward the center column of the array). The dithered images were then shifted and averaged using the DRIZZLE routine in IRAF (Fruchter \& Hook 1997). Finally, a background level of 0.45 analog-to-digital converter units (ADU) $\mathrm{s}^{-1}$ was measured from the blank-sky data, and this amount was subtracted from the $2.2 \mu \mathrm{m}$ image of NGC 4258 . The background level at 1.1 and $1.6 \mu \mathrm{m}$ was ignored since it is typically less than $0.01 \mathrm{ADU} \mathrm{s}^{-1}$. The plate scales of the final "drizzled" images, shown in Figure 1, are 0"0381 and 0"0378 pixel $^{-1}$ in $x$ and $y$.

Flux calibration of the images was done using scaling factors of $2.195 \times 10^{-6}, 2.207 \times 10^{-6}$, and $5.583 \times 10^{-6} \mathrm{Jy}$ $\left(\mathrm{ADU} \mathrm{s}^{-1}\right)^{-1}$ at 1.1, 1.6, and $2.2 \mu \mathrm{m}$ (M. J. Rieke et al. 1999, private communication). The corresponding magnitudes were calculated using the zero points 1909,1087 , and $665 \mathrm{Jy}$ at $0 \mathrm{mag}$ for $1.1,1.6$, and $2.2 \mu \mathrm{m}$, respectively. The 1.1, 1.6, and $2.2 \mu \mathrm{m}$ images were aligned to within 0.2 pixel using centroids and elliptical isophotes generated. Synthetic NICMOS point-spread functions (PSFs) were generated using the TINYTIM routine (Krist \& Hook 1997) since no observed PSFs were available.

As shown by CB97, the nucleus of the galaxy is clearly redder than the surrounding starlight. The colors of the nuclear regions $\left(\sim 00^{\prime \prime} 12\right.$ radius from the center $)$ are $[1.6]-[2.2]=0.9 \pm 0.1 \mathrm{mag}$ and $[1.1]-[1.6]=1.1 \pm 0.1$ mag. In comparison, the colors of the galaxy in an annulus of radius $0.5-1.0$ are $[1.6]-[2.2]=0.52 \pm 0.05 \mathrm{mag}$ and $[1.1]-[1.6]=0.91 \pm 0.05 \mathrm{mag}$. The reddening can be attributed to either a red central source or dust extinction of starlight. CB97 argued for the former, and we provide additional evidence in this paper that this is true. To estimate the contribution of the putative source seen by CB97 at the nucleus of the galaxy, it was necessary to subtract a stellar background from the galaxy frames. The $1.1 \mu \mathrm{m}$ surface brightness profile was fitted with an exponential and subtracted off such that the residual consisted of a central core with an FWHM corresponding to the FWHM of the $1.1 \mu \mathrm{m}$ PSF $\left(\sim 00^{\prime \prime} 1\right)$. There is no evidence of an Airy ring in the residual, and hence we conclude that we do not detect a compact component of emission at $1.1 \mu \mathrm{m}$. The residual has a flux density of $0.5 \mathrm{mJy}$, and we consider it an upper limit to a point source. 


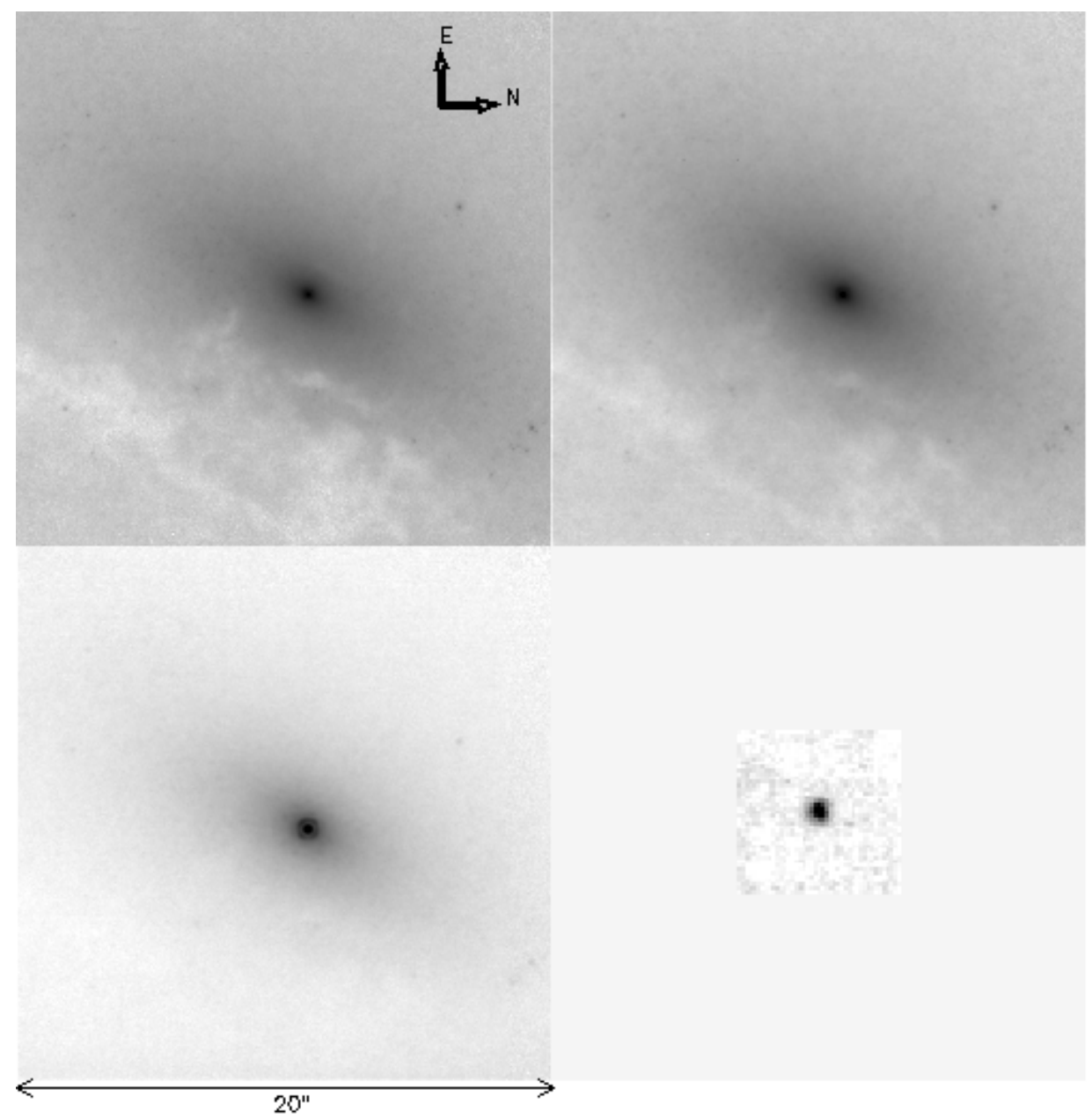

FIG. 1.-Morphology of the nuclear regions in NGC 4258 at (clockwise from upper left )1.1, 1.6, 12.5, and $2.2 \mu$ m. The near-infrared images are $20^{\prime \prime}$ on a side. The 1.1 and $1.6 \mu \mathrm{m}$ images clearly show the dust lane in the nuclear regions of the galaxy. The $12.5 \mu \mathrm{m}$ image, which is $6^{\prime \prime}$ on a side, does not show any extended emission. $\alpha=12^{\mathrm{h}} 18^{\mathrm{m}} 57.5^{\mathrm{s}}, \delta=47^{\circ} 18^{\prime} 14^{\prime \prime}(\mathrm{J} 2000)$.

Since the $1.1 \mu \mathrm{m}$ data do not show a compact source, it is reasonable to assume that they trace the distribution of starlight in the central regions of the galaxy. Furthermore, the contribution from the polarized component observed by W95, based on its flux density and spectrum, is found to be less than $0.1 \mathrm{mJy}$ at $1.1 \mu \mathrm{m}$. A scaled $1.1 \mu \mathrm{m}$ surface brightness image was subtracted from the 1.6 and $2.2 \mu \mathrm{m}$ surface brightness images. The scale factor was derived by performing an intensity-weighted least-squares fit within the central arcsecond of the form $\Sigma_{[2.2]}=a_{1} \Sigma_{[1.1]}+b_{1} P_{[2.2]}$, where $\sigma$ is the surface brightness, $a_{1}$ and $i_{1}$ are constants, and $P$ is the TINYTIM-generated PSF. For the $2.2 \mu \mathrm{m}$ image we obtain $a_{1}=1.25 \pm 0.05$, and for the $1.6 \mu \mathrm{m}$ image we get $a_{1}=1.29 \pm 0.05$. This corresponds to [1.1] $-[2.2]$ and $[1.1]-[1.6]$ colors of $1.39 \pm 0.05$ and $0.89 \pm 0.05 \mathrm{mag}$, respectively.

The Airy ring is clearly visible in the starlight-subtracted 1.6 and $2.2 \mu \mathrm{m}$ images that were obtained as described above. The FWHM of the residual is also very similar to the FWHM of the synthetic PSFs at the respective wavelengths. We thereby conclude that the compact component of emission at 1.6 and $2.2 \mu \mathrm{m}$ is unresolved at our resolution of $\sim 0$.'2. This implies that the size of the emitting region has a radius $\lesssim 3.5 \mathrm{pc}$ at the distance of the galaxy and is consistent with the emission originating from the vicinity of the black hole at the nucleus. The profile of the central source is shown in Figure 2. The brightness of the central source was measured by standard circular aperture photometry. The observed flux density of the compact source is $4.0 \pm 0.7 \mathrm{mJy}$ at $2.2 \mu \mathrm{m}$ and $0.9 \pm 0.3 \mathrm{mJy}$ at $1.6 \mu \mathrm{m}$. This agrees well with the values of 4.5 and $1.1 \mathrm{mJy}$ obtained by CB97 at $K$ and $H$, respectively.

M. J. Rieke (1999, private communication) has suggested that using the standard NICMOS calibration numbers for red objects, as above, could result in an error of as much as $0.7 \mathrm{mag}$ in the intrinsic NICMOS magnitude especially at [1.1]. The effect is smaller at [1.6] and negligible at [2.2]. This is because red objects would shift the effective wavelength and the zero point of the F110W and F160W filters, both of which have passbands much wider than the groundbased $J$ and $H$ filters. We estimate the amount of this error for NGC 4258 by applying a conversion from instrumental NICMOS magnitudes to ground-based magnitudes (see Appendix). This also serves a secondary purpose, which is to compare the colors of the starlight in NGC 4258, derived from the NICMOS data, with ground-based colors.

We have converted the NICMOS [1.1] and [1.6] images to ground-based $J$ and $H$ magnitudes and rederived the compact source flux density using the procedure described earlier. We find that the starlight in the galaxy has a $J-K$ 

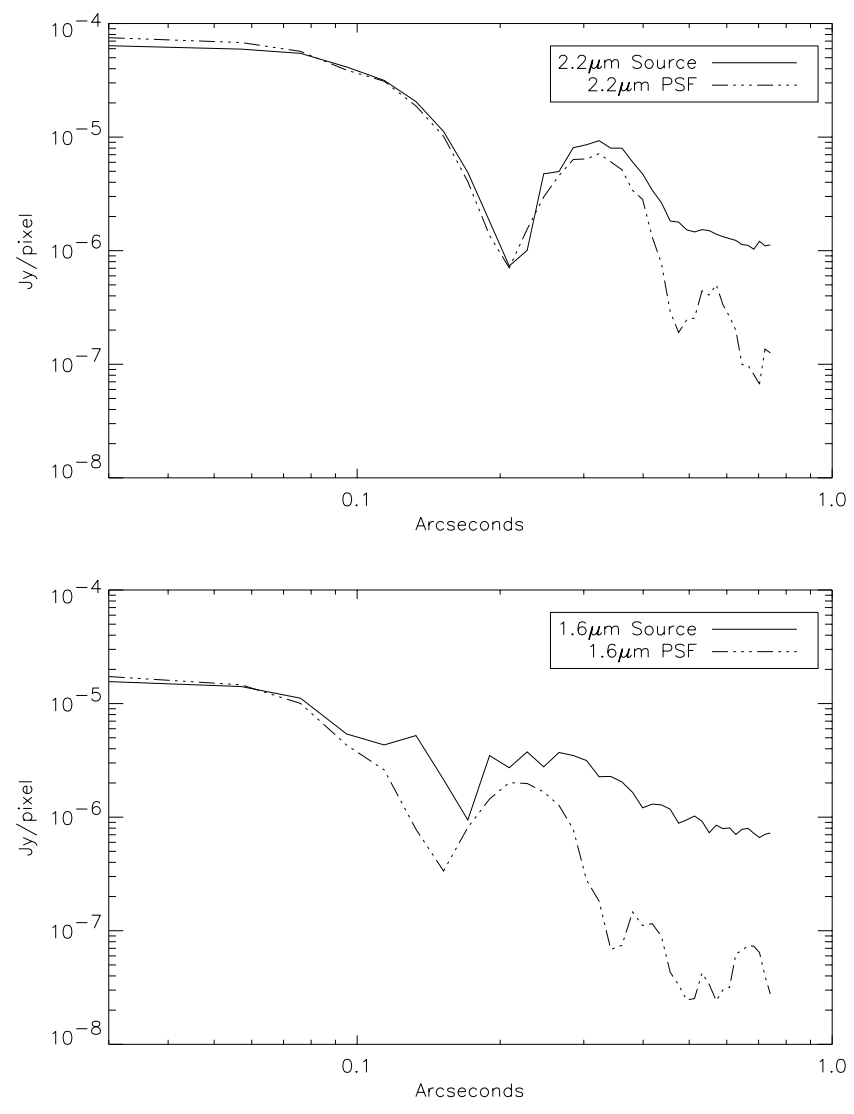

FIG. 2.-Profile of the central source at 2.2 and $1.6 \mu \mathrm{m}$ compared to a TINYTIM-generated point source.

color of $1.0 \mathrm{mag}$ and a $J-H$ color of $0.6 \mathrm{mag}$. This is in excellent agreement with the ground-based colors of $J-K=0.9 \mathrm{mag}$ and $J-H=0.6 \mathrm{mag}$ obtained by CB97 at a distance of 6 " from the center. These colors are consistent with the colors of late-type stars expected to dominate the surface brightness in the central regions of the galaxy. The resultant flux density of the central source is $1.1 \mathrm{mJy}$ at 1.6 $\mu \mathrm{m}$ and $3.6 \mathrm{mJy}$ at $2.2 \mu \mathrm{m}$. This is within the photometric errors assigned to the compact component above, and hence we conclude that the wide bandpass of the filters does not significantly affect our determination of the compact source flux density.

Near-infrared data were also obtained in the $L_{s}\left(\lambda_{0}=\right.$ $3.45 \mu \mathrm{m}, \Delta \lambda=0.57 \mu \mathrm{m})$ and $K_{s}\left(\lambda_{0}=2.15 \mu \mathrm{m}, \Delta \lambda=0.33\right.$ $\mu \mathrm{m})$ bands using the Cassegrain IR Camera on the $5.1 \mathrm{~m}$ Hale telescope. A $K_{s}$ PSF source was scaled to the flux density of the compact $2.2 \mu \mathrm{m}$ source in NGC 4258 and subtracted from the $K_{s}$ image of the galaxy after aligning. The residual image corresponds to the surface brightness profile of the starlight in the galaxy. This was then normalized to the $L_{s}$ surface brightness at a radius of 2 ".25 and subtracted. The normalization factor of $\Sigma_{K s} / \Sigma_{L s}=1.98$ corresponds to a $K_{s}-L_{s}$ color of $0.16 \mathrm{mag}$, which is reasonable considering the $J-H$ and $H-K$ colors of the starlight. Photometry was performed on the $L_{s}$ residual image, which is dominated by the compact component. We measure a flux density of $20 \pm 3 \mathrm{mJy}$ for this source.

The mid-infrared data were obtained using the MidInfrared Large Well Imager (MIRLIN) camera on the $10 \mathrm{~m}$ Keck II telescope in 1998 March (Ressler et al. 1994). The array is a $128 \times 128$ array with a pixel scale of $\sim 0$ ". 14 pixel $^{-1}$ at the $\mathrm{f} / 40$ bent Cassegrain focus of Keck II. The instrument has a square wave chopper that chops at $5 \mathrm{~Hz}$. The chopper throw was set at 6", which allowed the source to be on the array at both chop positions. Observations were made with a filter that is centered at $12.5 \mu \mathrm{m}$ with a passband of $1.2 \mu \mathrm{m}$ and a filter that is centered at $17.9 \mu \mathrm{m}$ with a passband of $2 \mu \mathrm{m}$. We chopped 300 times per position and nodded by $6^{\prime \prime}$ after a minute. The total integration time was $300 \mathrm{~s}$ at $12.5 \mu \mathrm{m}$ and $450 \mathrm{~s}$ at $17.9 \mu \mathrm{m}$. The resultant $1 \sigma$ sensitivity at 12.5 and $17.9 \mu \mathrm{m}$ was 20 and $50 \mathrm{mJy}$ in a 2 ".8 diameter beam, respectively.

The mid-infrared data were reduced in a standard manner (Bock et al. 1998). Air-mass corrections were performed that were less than $5 \%$ since the standards and source were at similar elevation angles. The final image at these wavelengths is dominated by a single unresolved source (FWHM $\lesssim 0$ ". 5 ) with no evidence of extended emission on scales of diameter up to 6 . The observed flux density of the unresolved central source is $165 \pm 20 \mathrm{mJy}$ at $12.5 \mu \mathrm{m}$ and $300 \pm 30 \mathrm{mJy}$ at $17.9 \mu \mathrm{m}$.

\section{EMISSION FROM A COMPACT EXTINCTED CONTINUUM SOURCE}

CB97 showed that the red near-infrared colors of the nucleus of the galaxy could not be explained by uniform foreground extinction of starlight or by stars mixed with dust. In addition, starlight cannot explain the observed spectrum of the enhanced nuclear emission at $\lambda>3 \mu \mathrm{m}$ (Table 1).

If the enhanced infrared emission in the nucleus arises in a compact nuclear continuum source associated with the $3.5 \times 10^{7} M_{\odot}$ black hole as argued by CB97, it implies that the extinction observed in the X-ray data occurs in a foreground screen such as a dust lane, attenuating both the near- and mid-infrared intensities. ${ }^{6}$ The visible/UV polarimetry data on the central source shows a $F_{v} \propto v^{-1.1 \pm 0.2}$ spectrum in scattered light (W95) similar to our observed mid-infrared spectrum. Therefore, it is reasonable to assume that the intrinsic near-infrared spectrum of the compact source follows a similar trend and that the observed [1.6] - [2.2] color of the compact source is due to extinction. We calculate that the amount of extinction required to fit the near- and mid-infrared data by a single power law corresponds to $A_{V}=18 \mathrm{mag}$. This is smaller than the extinction derived from the X-ray column density. However, as pointed out by Gammie, Narayan, \& Blandford (1999), the column density $N_{\mathrm{H}}$ derived by Makishima et al. (1994) is unreliable since it is sensitive to the relative contributions of the components used to fit the X-ray data. In addition, the conversions from the X-ray opacity, $\tau_{\mathrm{X}}$ to $N_{\mathrm{H}}$ and from $N_{\mathrm{H}}$ to $A_{V}$ are not well established. The resultant extinction-corrected infrared spectrum follows a $v^{-1.4 \pm 0.1}$ power law, suggesting a nonthermal origin. The extinction-corrected flux densities are shown in Figure 3 and Table 1.

The extrapolated flux density of the central source at 1.1 $\mu \mathrm{m}$ based on our fitted spectrum is calculated to be $10 \mathrm{mJy}$. If we extinct this $1.1 \mu \mathrm{m}$ source by $A_{V}=18 \mathrm{mag}$, we obtain a flux density of $0.1 \mathrm{mJy}$, consistent with our derived upper limit. The $0.6-20 \mu \mathrm{m}$ luminosity of the central source derived from our fitted $F_{v} \propto v^{-1.4}$ spectrum is $8.5 \times 10^{41}$

\footnotetext{
${ }^{6}$ For the rest of this paper, we assume $A_{[1.1]}=0.282 A_{V}, A_{[1.6]}=$ $0.624 A_{[1.1]}, A_{[2.2]}=0.382 A_{[1.1]}, A_{[3.5]}=0.182 A_{[1.1]}, A_{[10]}=0.192 A_{[1.1]}$, $A_{[12.5]}=0.098 A_{[1.1]}$, and $A_{[17.9]}=0.083 A_{[1.1]}($ Mathis 1990).
} 
TABLE 1

ENERGY Distribution FOR THE COMPACT SOURCE

\begin{tabular}{|c|c|c|c|c|}
\hline$\lambda$ & $\begin{array}{c}v \\
(\mathrm{~Hz})\end{array}$ & $\begin{array}{l}\text { Observed } F_{v} \\
(\mathrm{mJy})\end{array}$ & $\begin{array}{l}\text { Extinction-corrected } F_{v} \\
(\mathrm{mJy})\end{array}$ & Reference \\
\hline $2-10 \mathrm{keV} \ldots \ldots$ & $1.45 \times 10^{18}$ & $\ldots$ & $4.7 \times 10^{-4}$ & 1 \\
\hline $0.55 \mu \mathrm{m} \ldots \ldots .$. & $5.45 \times 10^{14}$ & $\ldots$ & $1.7-170$ & 2 \\
\hline $1.1 \mu \mathrm{m} \ldots \ldots \ldots$ & $2.73 \times 10^{14}$ & $<0.5$ & 10 & \\
\hline $1.6 \mu \mathrm{m} \ldots \ldots \ldots$ & $1.88 \times 10^{14}$ & $0.9 \pm 0.3$ & 16 & \\
\hline $2.2 \mu \mathrm{m} \ldots \ldots \ldots$ & $1.36 \times 10^{14}$ & $4.0 \pm 0.7$ & 25 & \\
\hline $3.45 \mu \mathrm{m} \ldots \ldots$. & $8.70 \times 10^{13}$ & $20 \pm 3$ & 46 & \\
\hline $10.5 \mu \mathrm{m}^{\mathrm{a}} \ldots \ldots$. & $2.86 \times 10^{13}$ & $100 \pm 12$ & 195 & 3 \\
\hline $12.5 \mu \mathrm{m} \ldots \ldots$. & $2.40 \times 10^{13}$ & $165 \pm 20$ & 270 & \\
\hline $17.9 \mu \mathrm{m} \ldots \ldots$. & $1.68 \times 10^{13}$ & $300 \pm 30$ & 435 & \\
\hline $1.4 \mathrm{~cm} \ldots \ldots \ldots$ & $22 \times 10^{9}$ & $\ldots$ & $<0.22$ & 4 \\
\hline
\end{tabular}

ergs $s^{-1}$. This is a factor of 20 greater than the X-ray luminosity.

RL78 measured a flux density of $100 \pm 12 \mathrm{mJy}$ in a 5.7 diameter beam with a $8-13 \mu \mathrm{m}$ filter. Since our mid-infrared images do not show any evidence of extended emission from the nucleus of the galaxy at scales of $6^{\prime \prime}$, this measurement must be dominated by the central source. If our extinctioncorrected measurements are interpolated to $10 \mu \mathrm{m}$ assuming a $v^{-1.4}$ spectrum, we obtain an unextincted flux density in the $8-13 \mu \mathrm{m}$ filter of $190 \mathrm{mJy}$. After putting in the extinction in the $8-13 \mu \mathrm{m}$ window including the $9.7 \mu \mathrm{m}$ silicate feature, corresponding to $A_{V}=18 \mathrm{mag}$, we derive an extincted $10 \mu \mathrm{m}$ flux density of $98 \mathrm{mJy}$ for the central source, in excellent agreement with the large-beam measurements.

The VLA observations of Turner \& Ho (1994) had detected the existence of a nonthermal source in the nucleus of the galaxy at 2 and $6 \mathrm{~cm}$. Milliarcsecond-resolution VLBI continuum observations indicate that most of the nonthermal radio emission arises in a subparsec-scale jet that is aligned with the rotation axis of the masing disk

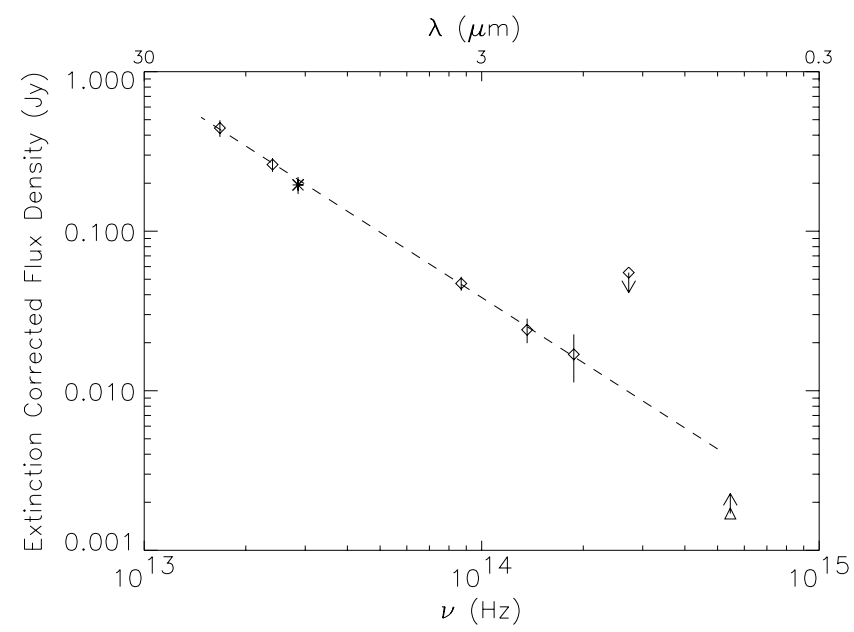

Fig. 3.-Fitted spectrum $\left(F_{v} \propto v^{-1.4}\right)$ of the central source along with the near- and mid-infrared data. These assume foreground extinction by a screen so both the near- and mid-infrared points have been extinction corrected. The visible light polarimetry measurement (triangle) of W95 is shown as a lower limit because of the large uncertainty in the value. The extinction-corrected RL78 measurement (asterisk) is shown.
(Herrnstein et al. 1997). The black hole region itself is undetected, and Herrnstein et al. (1998) obtain a $3 \sigma$ upper limit of $220 \mu \mathrm{Jy}$ at $22 \mathrm{GHz}$. When compared to the extinctioncorrected infrared spectrum, this seems to suggest that the spectrum has a turnover probably due to synchrotron selfabsorption between $20 \mu \mathrm{m}$ and $0.2 \mathrm{~mm}$. The total luminosity of the central source longward of $1 \mu \mathrm{m}$ is relatively insensitive to the turnover frequency and is constrained to lie between $8 \times 10^{41}$ and $3 \times 10^{42}$ ergs s $^{-1}$ for turnover wavelengths of 20 and $0.2 \mathrm{~mm}$, respectively. Spinoglio \& Malkan (1989) suggested that the $12 \mu \mathrm{m}$ luminosity is typically about $1 / 5$ of the bolometric luminosity of active galaxies, independent of whether the emission is thermal or nonthermal in origin. We find, by this argument, the bolometric luminosity of NGC 4258 to be $\sim 10^{42}$ ergs $\mathrm{s}^{-1}$, which is within our independently derived luminosity range.

As indicated by the extinction correction to our measurements, the nucleus of NGC 4258 clearly has dust associated with it. The extinction-corrected infrared emission from 1 to $20 \mu \mathrm{m}$ has a $v^{-1.4}$ power-law spectrum that appears to extend up to visible wavelengths as seen in the polarimetric observations of W95. This makes thermal dust emission at these wavelengths unlikely, and we infer that the infrared emission is nonthermal in origin. Many previous authors have concluded that the source of both the near- and midinfrared emission in the nuclei of Seyfert galaxies is thermal emission from dust reprocessing of a blue, nuclear continuum (e.g., Giuricin, Mardirossian, \& Mezzetti 1995). This was based on a weak correlation between the X-ray and mid-infrared emission in a large sample of Seyfert galaxies. Our derived spectrum suggests that it is more likely that the dust in the nucleus of NGC 4258 is cool and contributes substantially only at far-infrared/submillimeter wavelengths.

\section{THE ACCRETION FLOW IN NGC 4258}

\subsection{Implications for an Advection-dominated Flow}

M97 has derived the $10^{8}-10^{20} \mathrm{~Hz}$ spectrum of systems dominated by an ADAF (Fig. 4). The spectrum consists of three components, a synchrotron component at $v<10^{12}$ $\mathrm{Hz}$, a power-law component for $10^{12}<v<10^{17} \mathrm{~Hz}$ from the inverse Compton scattering of the cyclosynchrotron photons, and a bremsstrahlung component at higher frequencies. 


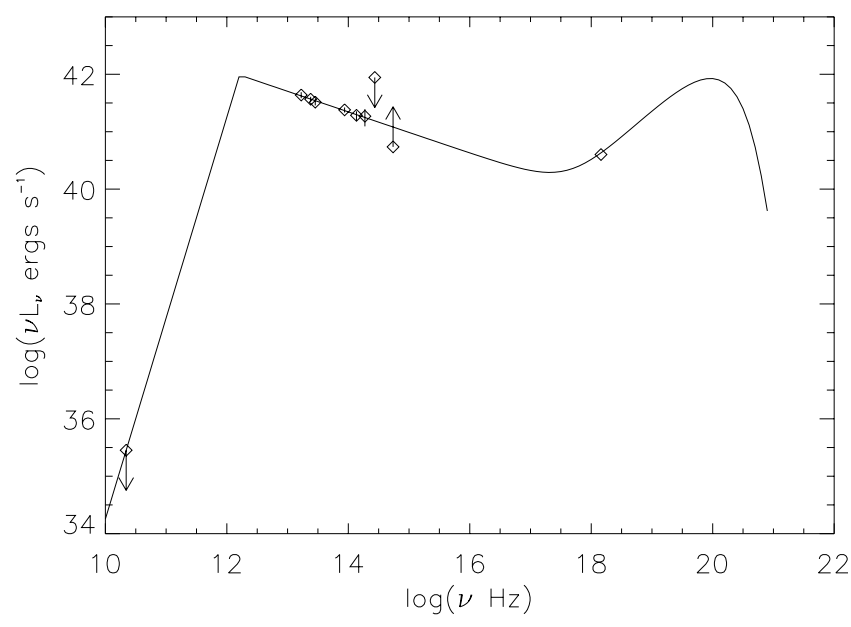

FIG. 4.-Spectrum of an ADAF fit to the data on NGC 4258. There are three components of emission, an $L_{v} \propto v^{5 / 2}$ synchrotron component, a $L_{v} \propto v^{-1.4}$ power-law component from Compton upscatter of the synchrotron photons, and a bremsstrahlung component with an exponential cutoff at frequencies $h v \geq k T_{e}$. The components from $v>10^{12} \mathrm{~Hz}$ agree with M97. Below that we have adopted a $v^{5 / 2}$ synchrotron component instead of the $v^{2 / 5}$ predicted in M97. The W95 visible light polarimetry is shown as a lower limit because of the large uncertainty in the measurement. The 1.1 $\mu \mathrm{m}$ upper limit derived from our observations, the $22 \mathrm{GHz}$ upper limit of Herrnstein et al. (1998), and the Makishima et al. (1994) X-ray measurement are also shown.

From M97, the spectral index of the power law $\left(L_{v} \propto v^{-\gamma}\right)$ is given by

$$
\begin{gathered}
\gamma \equiv \frac{-\ln \tau_{\mathrm{es}}}{\ln A}, \\
\tau_{\mathrm{es}}=(23.87 \dot{m})\left(\frac{0.3}{\alpha}\right)\left(\frac{0.5}{c_{1}}\right)\left(\frac{3}{r_{\min }}\right)^{1 / 2},
\end{gathered}
$$

and

$$
A=1+4 \theta_{e}+16 \theta_{e}^{2},
$$

where $\tau_{\text {es }}$ is the optical depth to electron scattering, $A$ is the mean photon energy amplification factor in a single scattering, $\theta_{e}=\left(k T_{e}\right) /\left(m_{e} c^{2}\right), c_{1}=0.5$ is a term relating the radial velocity of the accretion flow to the Keplerian velocity, and $r_{\min }$ is the inner radius of the ADAF, which we assume to be $3 R_{\mathrm{S}} . T_{e}$ is the electron temperature, which is constant over the ADAF region. Based on the figures from M97, we adopt $T_{e}=4.4 \times 10^{9} \mathrm{~K}, \alpha=0.3$ and derive from the above equations an accretion rate of $\dot{m} \sim 1.2 \times 10^{-3}$ in Eddington units, which corresponds to $\dot{M} \sim 9 \times 10^{-4} M_{\odot}$ $\mathrm{yr}^{-1}$.

It is also possible to estimate $\dot{m}$ from the bolometric luminosity as shown by M97. For $\alpha=0.3, \beta=0.5$, we find that our $L_{\mathrm{Bol}} \sim 10^{42} \mathrm{ergs} \mathrm{s}^{-1}$ corresponds to $\dot{m} \sim 6 \times 10^{-3}$ in Eddington units, which is again reasonable considering the factor of 3 uncertainty in the bolometric luminosity. $\beta$ is the ratio of gas pressure to total pressure.

In comparison, Lasota et al. (1996) derived a rate of $\dot{m} \sim$ $0.16 \alpha$ through an ADAF. However, at that time the infrared data that better constrain the bolometric luminosity of the central engine were not available, and the ADAF models were fitted to the slope of the X-ray data. More recently, Gammie et al. (1999) derive an accretion rate of $0.01 M_{\odot} \mathrm{yr}^{-1}$ through an ADAF using the infrared data of CB97 as a constraint. Again, this analysis assumed that most of the
visible/IR emission has a $v^{1 / 3}$ Comptonized blackbody spectrum and arises in the cool, thin disk rather than in the ADAF itself. Our recent data suggests that the visible/IR emission has a common origin and has an intrinsic spectrum that can be well represented by a $\sim v^{-1}$ power law.

Herrnstein et al. (1998) have shown, based on their 22 $\mathrm{GHz}$ upper limit, that the ADAF cannot extend significantly beyond $\sim 10^{2} R_{\mathrm{S}}$. We find that the $22 \mathrm{GHz}$ limit in conjunction with the spectrum of M97 in the self-absorbed radio regime is inconsistent with our measurements (Fig. 4). We predict that the radio synchrotron spectrum must have a spectral index of $2.5 \geq \dot{\gamma} \geq 1.2$, where $F_{v} \propto v^{\gamma}$ rather than the $\dot{\gamma}=2 / 5$ derived for a thermal distribution of electrons by M97. This could arise if the distribution of electrons has a power-law tail at higher energies. Maintaining a powerlaw distribution of relativistic particles without resorting to an independent injection mechanism such as magnetic reconnection has been an unsolved problem for some time now (McCray 1969). All previous work has assumed that the particle distribution in an ADAF is thermal. However, Mahadevan \& Quataert (1997) investigated the emission spectrum for different accretion rates through an ADAF. They found that, for low accretion rates, the thermalization of the electron distribution through Coulomb collisions with the protons and through self-absorption of synchrotron photons is inefficient. Adiabatic compression of the electron gas acts as the primary heating mechanism, which results in the high-energy tail of the electron distribution function having a nonthermal Gaussian profile rather than a Maxwellian profile. Alternatively, Mahadevan, Narayan, \& Krolik (1997) have suggested that if the weak Galactic center source $\mathrm{Sgr} \mathrm{A}^{*}$ is associated with the EGRET source 2EG J1746-2852, the gamma-ray spectrum could be explained by an $E^{-2.75}$ distribution of protons in an ADAF. A similar power-law distribution of electrons would indicate that the electron distribution function is generated by the same viscous heating mechanism responsible for generating the proton distribution function.

\subsection{Implications for a Standard Thin Disk}

We find that the excellent agreement between the fit $F_{v} \propto v^{-1.4}$ spectrum and data obviates the need for a significant $v^{1 / 3}$ Comptonized blackbody continuum from a standard optically thick, geometrically thin disk. However, $22 \mathrm{GHz}$ observations show the existence of a thin disk with inner radius $0.14 \mathrm{pc}$ that sustains the water masers (Miyoshi et al. 1995). From standard disk theory (Frank, King, \& Raine 1992),

$$
T_{\text {eff }} \sim 3.5 \times 10^{7} m^{-1 / 4} \dot{m}^{1 / 4} r^{-3 / 4} \mathrm{~K},
$$

where $m$ is the central mass in units of $M_{\odot}$ and $R$ is the radius of the disk in units of $R_{\mathrm{S}}$. For a geometrically thin, optically thick disk to be capable of emitting blackbody near-infrared photons, it should be at least $1500 \mathrm{~K}$. Based on the derived accretion rate, this implies a conservative lower limit of $200 R_{\mathrm{S}}$ for the inner radius of the thin disk.

\section{CONCLUSIONS}

NGC 4258 has associated with it a compact nuclear source at near- and mid-infrared wavelengths that is unresolved even at a near-infrared resolution of $\sim 0$ ". 2 FWHM. This implies that the emitting region is $\lesssim 3.5 \mathrm{pc}$ in radius at the distance of the galaxy. The observed infrared flux densities can be fitted by a single power law of the form $F_{v} \propto$ 

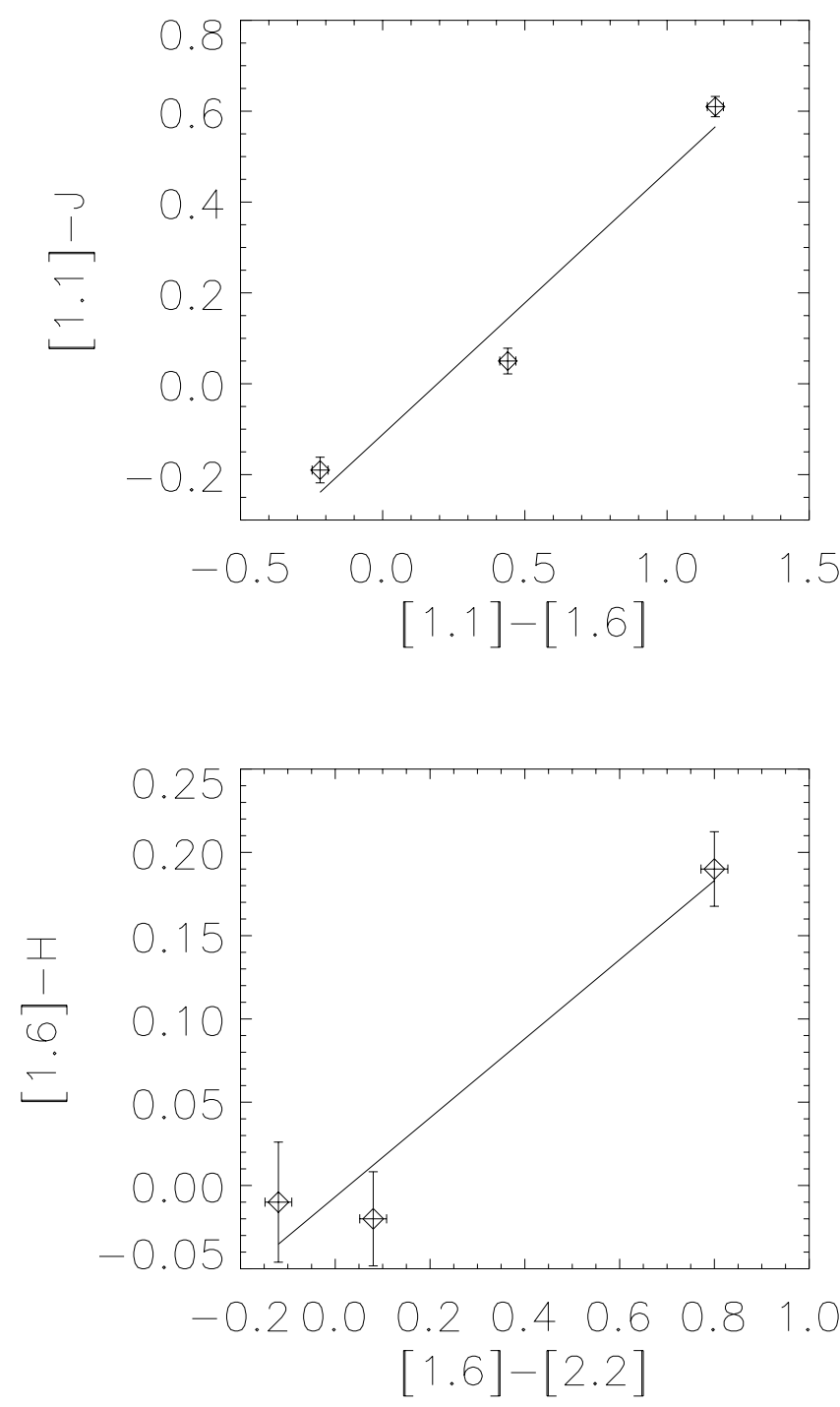

FIG. 5.-Color correction between NICMOS magnitudes and groundbased magnitudes based on observations of standards of different colors. The lines are the best-fit first-order polynomials described in the text. The color correction at [2.2] is small enough that we take it to be 0 . $v^{-1.4 \pm 0.1}$ from 1 to $18 \mu \mathrm{m}$ after correcting for about $18 \mathrm{mag}$ of visual extinction. The total extinction-corrected infrared luminosity $(1-20 \mu \mathrm{m})$ of the central source is $8 \times 10^{41} \mathrm{ergs}$ $\mathrm{s}^{-1}$. At shorter wavelengths, the power-law spectrum seems to extend up to visible wavelengths as shown by the polarimetric observations of W95. At longer wavelengths, the infrared spectrum along with the $22 \mathrm{GHz}$ upper limit points toward a synchrotron self-absorption turnover wavelength between $20 \mu \mathrm{m}$ and $0.2 \mathrm{~mm}$. From this spectrum, we derive the bolometric luminosity of the nuclear source to be $\sim 2 \times 10^{42} \mathrm{ergs} \mathrm{s}^{-1}$. The low bolometric luminosity and power-law spectrum extending from visible to mid-infrared wavelengths indicates that self-Comptonized synchrotron emission arising in an ADAF is responsible for the enhanced infrared emission. The spectrum obviates the need for a "big blue bump" at these wavelengths from a cool, optically thick, geometrically thin disk extending beyond the advection-dominated flow. Such a disk is predicted to have a $F_{v} \propto v^{1 / 3}$ spectrum, which appears unlikely from our data. Based on the spectrum of the source and the derived bolometric luminosity, we infer the accretion rate through an ADAF in NGC 4258 to be $\sim 10^{-3} M_{\odot} \mathrm{yr}^{-1}$.

This work is based on observations made at the W. M. Keck Observatory, the Palomar Observatory, and with the NASA/ESA Hubble Space Telescope, which is operated by the Association of Universities for Research in Astronomy, Inc., under NASA contract NAS5-26555. We wish to acknowledge the contributions made by members of the NICMOS Instrument Definition Team to this project. We are grateful to Steve Willner for his help with clarifying the discussion in the Appendix. We would also like to thank the staffs of the respective observatories for their assistance. The W. M. Keck Observatory is a scientific partnership between the University of California and the California Institute of Technology.

\section{APPENDIX \\ CONVERSION OF NICMOS MAGNITUDES TO GROUND-BASED MAGNITUDES}

In this section, we describe the color correction applied to convert the NICMOS [1.1], [1.6], and [2.2] magnitudes to ground-based $J, H$, and $K$ magnitudes. The color correction is applied for two reasons: (1) to compare the results from this NICMOS data set with previous ground-based observations and (2) to correct for calibration errors that arise as a result of the wider bandpass of the NICMOS F110W and F160W filters. It has been suggested that since the NICMOS filters were calibrated off solar analog stars, using the standard NICMOS calibration numbers could result in an error of as much as 0.7 mag in the intrinsic NICMOS magnitude for red $(J-H>1 \mathrm{mag}$ ) objects (M. J. Rieke 1999, private communication). This error is not significant in the ground-based $J$ and $H$ filters because of their much narrower bandpass.

TABLE 2

PHOTOMETRY OF UNEXTINCTED NICMOS STARS ${ }^{\mathrm{a}}$

\begin{tabular}{ccccccc}
\hline \hline Object & {$[1.1]$} & $J$ & {$[1.6]$} & $H$ & {$[2.2]$} & $K$ \\
\hline G191-B2B (WD) $\ldots \ldots .$. & $12.49 \pm 0.02$ & $12.68 \pm 0.02$ & $12.71 \pm 0.02$ & $12.72 \pm 0.03$ & $12.83 \pm 0.02$ & $\ldots$ \\
P330E (G star) $\ldots \ldots .$. & $12.01 \pm 0.02$ & $11.96 \pm 0.02$ & $11.57 \pm 0.02$ & $11.59 \pm 0.02$ & $11.49 \pm 0.02$ & $11.51 \pm 0.02$ \\
BRI 0021 (red) $\ldots . . . .$. & $12.45 \pm 0.02$ & $11.84 \pm 0.01$ & $11.28 \pm 0.02$ & $11.09 \pm 0.01$ & $10.48 \pm 0.02$ & $10.55 \pm 0.01$ \\
\hline
\end{tabular}

${ }^{\text {a }}$ Obtained from the STScI Web site with the errors not representing calibration errors. 
NICMOS has observed a sample of standards of different colors for which the ground-based magnitudes are known (Table 2). Thus, by converting instrumental magnitudes to ground-based magnitudes on the basis of these observations, we can eliminate calibration errors. Figure 5 shows the color correction with the best corresponding linear fit listed below. The color correction at [2.2] is small enough that we take it to be 0 .

$$
\begin{aligned}
J-[1.1] & =-0.58 \times([1.1]-[1.6])+0.11 \\
H-[1.6] & =-0.24 \times([1.6]-[2.2])+0.01 .
\end{aligned}
$$

\section{REFERENCES}

Bock, J. J., Marsh, K. A., Ressler, M. E., \& Werner, M. W. 1998, ApJ, 504, L5

Bushouse, H. 1997, in Proc. STScI Workshop (1997 September) The 1997 HST Calibration Workshop with a New Generation of Instruments, ed. S. Casertano (Baltimore: STScI), 223

Chary, R., \& Becklin, E. E. 1997, ApJ, 485, L75 (CB97)

Filippenko, A. V. 1996, in ASP Conf. Ser. 103, The Physics of LINERs in View of Recent Observations, ed. M. Eracleous et al. (San Francisco: ASP), 17

Frank, J., King, A. R., \& Raine, D. 1992, Accretion Power in Astrophysics (Cambridge: Cambridge Univ. Press)

Fruchter, A., \& Hook, R. N. 1997, ST-ECF Newsl., 24, 9

Gammie, C. F., Narayan, R., \& Blandford, R. D. 1999, ApJ, 516, 177

Genzel, R., Eckart, A., Ott, T., \& Eisenhauer, F. 1997, MNRAS, 291, 219

Ghez, A. M., Klein, B. L., Morris, M., \& Becklin, E. E. 1998, ApJ, 509, 678

Giuricin, G., Mardirossian, F., \& Mezzetti, M. 1995, ApJ, 446, 550

Herrnstein, J. R., Greenhill, L. J., \& Moran, J. M. 1996, ApJ, 468, L17

Herrnstein, J. R., Greenhill, L. J., Moran, J. M., Diamond, P. J., Inoue, M., Nakai, N., \& Miyoshi, M. 1998, ApJ, 497, L69

Herrnstein, J. R., Moran, J. M., Greenhill, L. J., Diamond, P. J., Miyoshi, M., Nakai, N., \& Inoue, M. 1997, ApJ, 475, L17

Herrnstein, J. R., et al. 1999, Nature, 400, 539

Krist, J., \& Hook, R. N. 1997, ST-ECF Newsl., 24, 10

Lasota, J. P., Abramowicz, M. A., Chen, X., Krolik, J., Narayan, R., \& Yi, I. 1996, ApJ, 462, 142
Mahadevan, R. 1997, ApJ, 477, 585 (M97)

Mahadevan, R., Narayan, R., \& Krolik, J. 1997, ApJ, 486, 268

Mahadevan, R., \& Quataert, E. 1997, ApJ, 490, 605

Makishima, K., et al. 1994, PASJ, 46, L77

Mathis, J. S. 1990, ARA\&A, 28, 37

McCray, R. 1969, ApJ, 156, 329

Miyoshi, M., Moran, J. M., Herrnstein, J. R., Greenhill, L. J., Nakai, N., Diamond, P. J., \& Inoue, M. 1995, Nature, 373, 127

Mushotzky, R. F., Done, C., \& Pounds, K. A. 1993, ARA\&A, 31, 717

Narayan, R., \& Yi, I. 1995, ApJ, 452, 710

Pringle, J. E. 1981, ARA\&A, 19, 137

Ressler, M. E., Werner, M. W., Van Cleve, J., \& Chou, H. A. 1994, in Infrared Astronomy with Arrays: The Next Generation, ed. I. S. McLean (Dordrecht: Kluwer), 429

Rieke, G. H., \& Lebofsky, M. J. 1978, ApJ, 220, L37 (RL78)

Scoville, N. Z., et al. 2000, AJ, in press

Shakura, N. I., \& Sunyaev, R. A. 1973, A\&A, 24, 337

Spinoglio, L., \& Malkan, M. A. 1989, ApJ, 342, 83

Thompson, R., Rieke, M., Schneider, G., Hines, D. C., \& Corbin, M. R. 1998, ApJ, 492, L95

Turner, J. L., \& Ho, P. T. P. 1994, ApJ, 421, 122

Wilkes, B. J., Schmidt, G. D., Smith, P. S., Mathur, S., \& McLeod, K. K. 1995, ApJ, 455, L13 (W95) 